\title{
Physical Activity and Cardiovascular Risk Factors in Children: a Meta-Analysis Update
}

Claudia Ciceri Cesa, ${ }^{1 \oplus}$ Gabriela Oliveira Gonçalves Molino, ${ }^{2 \oplus}$ Júlia Lima, ${ }^{\circledR}$ Raquel Barbiero Pereira, ${ }^{4 \oplus}$ Bruna Eibel, $^{3 \oplus}$ Sandra Mari Barbiero, ${ }^{3 \oplus}$ Beatriz D. Schaan, ${ }^{4,5}{ }^{\oplus}$ Lucia Campos Pellanda $^{2,3}$

Instituto Federal Sul-rio-grandense - DEPEP,' Sapucaia do SUI, RS-Brazil

Universidade Federal de Ciências da Saúde de Porto Alegre, ${ }^{2}$ Porto Alegre, RS - Brazil

Instituto de Cardiologia - Fundação Universitária de Cardiologia, ${ }^{3}$ Porto Alegre, RS - Brazil

Universidade Federal do Rio Grande do Sul, ${ }^{4}$ Porto Alegre, RS - Brazil

Hospital de Clínicas de Porto Alegre, ${ }^{5}$ Porto Alegre, RS - Brazil

\section{Abstract}

Background: Obesity and overweight in childhood can increase the risk of developing cardiovascular disease throughout live.

Objectives: This study provides an update of a meta-analysis of randomized clinical trials (RCT) published in 2014, to assess the effects of physical activity interventions on preventing cardiovascular risk factors in childhood.

Methods: This update combines data from the previous search with new data obtained from June 2013 to June 2020. Searches were performed on PubMed, EMBASE and Cochrane CENTRAL. The RCTs enrolled used interventions with physical activity longer than six months in school children aged 6-12 years, and evaluated body mass index (BMI), systolic blood pressure (SBP), diastolic blood pressure (DBP), total cholesterol (TC), triglyceride (TG), and low-density lipoprotein (LDL) and high-density lipoprotein (HDL) levels. Data analysis was performed using a random-effects model and a P value $<0.05$ was considered statistically significant.

Results: A total of 28,603 articles were retrieved, and 17 RCTs (11,952 subjects) were included. Physical activity interventions were associated with reduction in SBP [-2.11 $\left.\mathrm{mmHg}(95 \% \mathrm{CI}-3.67,-0.54), \mathrm{I}^{2} 43 \%\right]$, DBP [-2.08mmHg (95\%CI $\left.-3.68,-0,49), \mathrm{I}^{2} 65 \%\right]$ and TG $\left[-0.08 \mathrm{mmol} / \mathrm{L}(95 \%\right.$ CI $\left.-0.13,-0.03), \mathrm{I}^{2} 0 \%\right]$, and increase in TC [0.17mmol/L $(95 \%$ CI $\left.0.04,0.30), \mathrm{I}^{2} 0 \%\right]$. However, the interventions were not associated with reductions in BMI $\left[-0.03 \mathrm{~kg} / \mathrm{m}^{2}\right.$ (95\%CI -0.17, 0.10), [20\%].

Conclusion: This update confirms and reinforces the beneficial effects of physical activity intervention in reducing systolic and diastolic blood pressure and TG levels.

Keywords: Children; Cardiovascular Diseases; Risk Factors; Physical, Activity Exercise, Public Health, Stress Test, Sports.

\section{Introduction}

The prevalence of overweight and obesity in childhood has increased in the last decade, being characterized as an alarming problem in developed and developing countries. ${ }^{1,2}$ Obesity is an important public health challenge, and its long-term consequences are well established. ${ }^{3}$ Overweight and obese children are likely to become obese adults. Also, they are at higher risk for developing chronic diseases such as high blood pressure, type 2 diabetes, cardiovascular disease, obstructive sleep apnea, breathing disorders, osteoarthritis and cancer. ${ }^{4,5}$

Thus, interventions during childhood might be beneficial to prevent these risks. The regular practice of physical activity in the long term is associated with improvement in body composition, with reduction of total and central adiposity, which contributes to a better blood pressure control, improvement of lipid and cardiorespiratory profiles, ${ }^{6,7}$ and prevention of several chronic diseases. ${ }^{8}$ Additionally, there is a

\section{Mailing Address: Claudia Ciceri Cesa}

Instituto Federal de Educação, Ciência e Tecnologia Sul-Rio-Grandense - IFSul Campus Sapucaia do Sul

Avenida Copacabana, 100. Postal Code: 93216-120, Sapucaia do Sul, RS - Brazil.

Email: claudia.c.cesa@gmail.com 
growing body of scientific evidence that recommends the practice of physical activity and points out the risks of sedentary behaviors. ${ }^{9}$

In a previous meta-analysis published in $2014{ }^{10}$ our group demonstrated that physical activity was associated with a reduction in triglycerides and blood pressure levels in school children and suggested that physical activity programs aiming at cardiovascular prevention should be stimulated in this population. Since the publication of this review, other clinical trials have been published. Therefore, this update aims to discuss recent evidence of mid-term effects of physical activity.

\section{Methods}

This systematic review is an update of the review published in 2014. All included studies in the 2014 review were considered in this update, in addition to studies published between 01 June 2013 and 15 June 2020. Details of our previous systematic review can be found in Cesa et al., ${ }^{10}$ all steps were performed according to the Cochrane Collaboration ${ }^{11}$ and the Preferred Reporting Items for Systematic Review and Meta-analyses: the PRISMA Statement. ${ }^{12}$

\section{Eligibility criteria}

Eligible studies were randomized clinical trials (RCTs) with students aged from 6 to 12 years old, submitted to a single or main intervention of supervised physical activity. Population criteria were school children irrespective of body weight (normal-weight, overweight and obese). Interventions were exercises with a minimum of 150 minutes per week for at least 6 months. A control group with no intervention or undergoing lower-intensity exercise (e.g., ordinary physical education classes with a duration of less than 150 minutes per week) should be included. A minimum target of 150 minutes per week was chosen as it is the amount of physical activity a person should engage in to be considered physically active. ${ }^{13,14}$ The variables included in the protocol were risk factors for the development of cardiovascular disease: systolic and diastolic blood pressure (SBP and $\mathrm{DBP}$, respectively), body mass index (BMI), total cholesterol (TC), low-density lipoprotein cholesterol (LDL-c), high-density lipoprotein cholesterol (HDL-c), fasting glucose and C-reactive protein (CRP).
To be included in the protocol, the RCT should meet the inclusion criteria and have at least one of the primary or secondary outcomes. The authors whose articles did not present the size of the intervention effect in the experimental and control group were contacted by e-mail in the attempt of obtaining missing information. In case of unsuccess, the studies were excluded. Studies with nutritional intervention were included only if the physical activity was the main intervention. For articles from multiple publications, the most recent publication was included, the previous ones were used for complementary information.

\section{Information sources}

The online databases searched were MEDLINE (accessed by PubMed), EMBASE and the Cochrane Central Register of Controlled Trials (CENTRAL). The following terms were used: "obesity", "overweight", "child nutrition disorders", "child", "school", "student", "exercise", "exercise therapy", "exercise movement techniques", "motor activity", "sports", "physical education and training", in addition to a highly sensitive strategy for the search for randomized clinical trials. ${ }^{15}$ The search strategy for PUBMED is shown in Appendix I. The authors searched and verified reference lists of systematic reviews and previously published metaanalysis to identify primary studies. This review had no language restrictions.

\section{Study selection and data extraction}

For this update, titles and abstracts of all articles identified by the search strategy were evaluated by three independent researchers in duplicate (B.E, C.C.C. and S.M.B). Those abstracts that did not provide enough information about inclusion and exclusion criteria were selected for a full-text evaluation. Disagreements between reviewers were evaluated by a third reviewer and solved by consensus (L.C.P). The outcomes extracted were: BMI $\left(\mathrm{Kg} / \mathrm{m}^{2}-\right.$ weight in kilograms divided by the square of the height in meters), SBP and DBP $(\mathrm{mmHg}), \mathrm{TC}$ and triglycerides (TG) (mmol/L), HDL-c and LDL-c (mmol/L).

\section{Assessment of risk of bias}

To assess the internal quality of the studies, we evaluated each RCT according to the Cochrane Collaboration's tool for assessing risk of bias. ${ }^{16}$ To be considered of good quality, the studies needed to present 
a clear description of the generation of suitable sequences, including description of the allocation concealment, blinding of the researcher, participants, assessors and evaluators of results, analysis of intention-to-treat and description of losses and exclusions. Concealed allocation was considered if the terms were described as "central", "web-based" or "telephone randomization", or full explanation. For the intention-to-treat analysis, the number of randomized participants and the number of patients analyzed should be the same; exceptions were patients who were lost to follow-up or asked to leave the study (they withdrew their consent). Three reviewers independently performed a quality assessment and, for each criterion, the studies were classified as adequate, not adequate or unclear/unreported.

\section{Data analysis}

All analyses were conducted using Review Manager version 5.3 (Cochrane Collaboration). For continuous outcomes, if the unit of measurement was consistent across trials, results were presented as weighted mean difference, with $95 \%$ confidence intervals (CIs). Pooled-effect estimates were obtained using the final values. ${ }^{17}$ Calculations were performed using a random-effect model and the statistical method used was inverse variance. Statistical heterogeneity of the treatment effects among studies was assessed using the Cochran's $\mathrm{Q}$ test and the inconsistency $\mathrm{I}^{2}$ test, and values above $25 \%$ and $50 \%$ were considered indicative of moderate and high heterogeneity, respectively. ${ }^{18}$

Sensitivity analysis was performed to evaluate differences in the intervention approach (intervention group: physical education classes + physical exercise program; control group: physical education classes or other types of intervention approaches). Heterogeneity between studies was also evaluated in terms of intensity and duration of the intervention and follow-up. A p-value $<0.05$ was considered statistically significant. Funnel plots were constructed to assess the risk of publication bias (Appendix II).

\section{Results}

\section{Description of studies}

In our previous systematic review, ${ }^{10} 23,091$ potentially relevant citations were retrieved and from June 2014 to June 2020 another 5,512 articles were identified (total 28,603).
Six studies met the inclusion criteria, which were added to the previous 11 selected, ${ }^{10}$ resulting in 17 studies included in this paper. Figure 1 shows the diagram flow of the studies in this review. Table 1 summarizes the characteristics of all studies (Appendix III).

\section{Risk of bias}

Of the six included studies, $47 \%$ had adequate sequence generation, $35 \%$ reported adequate allocation concealment, $29 \%$ reported adequate blinding of clinical assessors, $71 \%$ performed intention-to-treat analyses and $94 \%$ described losses to follow-up exclusions. Table 2 describes the risk of bias of the studies (Appendix IV).

\section{Effects of the interventions}

\section{Body mass index}

Of 17 studies, 13 [Vandongen et al., ${ }^{19}$ Barbeau et al., ${ }^{20}$ Donnelly et al., ${ }^{21}$ Kriemler et al., ${ }^{22}$ Li et al., ${ }^{23}$ Faude et al., ${ }^{24}$ Jansen et al., Thivel et al., ${ }^{26}$ Weintraub et al., ${ }^{27}$ Martínez-Vizcaíno et al., ${ }^{28}$ Naiman et al., ${ }^{29}$ Jones et al., ${ }^{30}$ Sacchetti et al., ${ }^{31} \mathrm{n}=11,952$, evaluated BMI. Physical activity interventions were not associated with changes in BMI when compared to less intensive physical activity interventions or no intervention (Figure 2), similarly to what was observed previously. ${ }^{10}$

\section{Lipid profile}

\section{Total cholesterol, cholesterol fractions and triglycerides}

Three studies [Vandongen et al., ${ }^{19}$ Walther et al., ${ }^{32}$ Jones et al., ${ }^{30}, \mathrm{n}=499$, evaluated TC. Physical activity interventions were associated with TC increase when compared to less intensive or no intervention (Figure 3), which is not different from previous findings. ${ }^{10}$ Two studies, Jones et al., ${ }^{30}$ Muller et al., ${ }^{33}$ evaluated LDL-c $(n=287)$ and HDL-c $(n=328)$. Physical activity interventions were not associated with changes in LDL-c or in HDL-c when compared to less intensive or no intervention (Figure 3). Triglycerides were evaluated in four [Kriemler et al., ${ }^{22}$ Walther et al., ${ }^{32}$ Jones et al., 2015; ${ }^{30}$ Muller et al., ${ }^{33}$ studies ( $\mathrm{n}=981$ ). Physical activity interventions were associated with reductions in TG when compared to less intensive physical activity interventions or no intervention, similarly to what was previously shown. ${ }^{10}$ (Figure 3 ) 


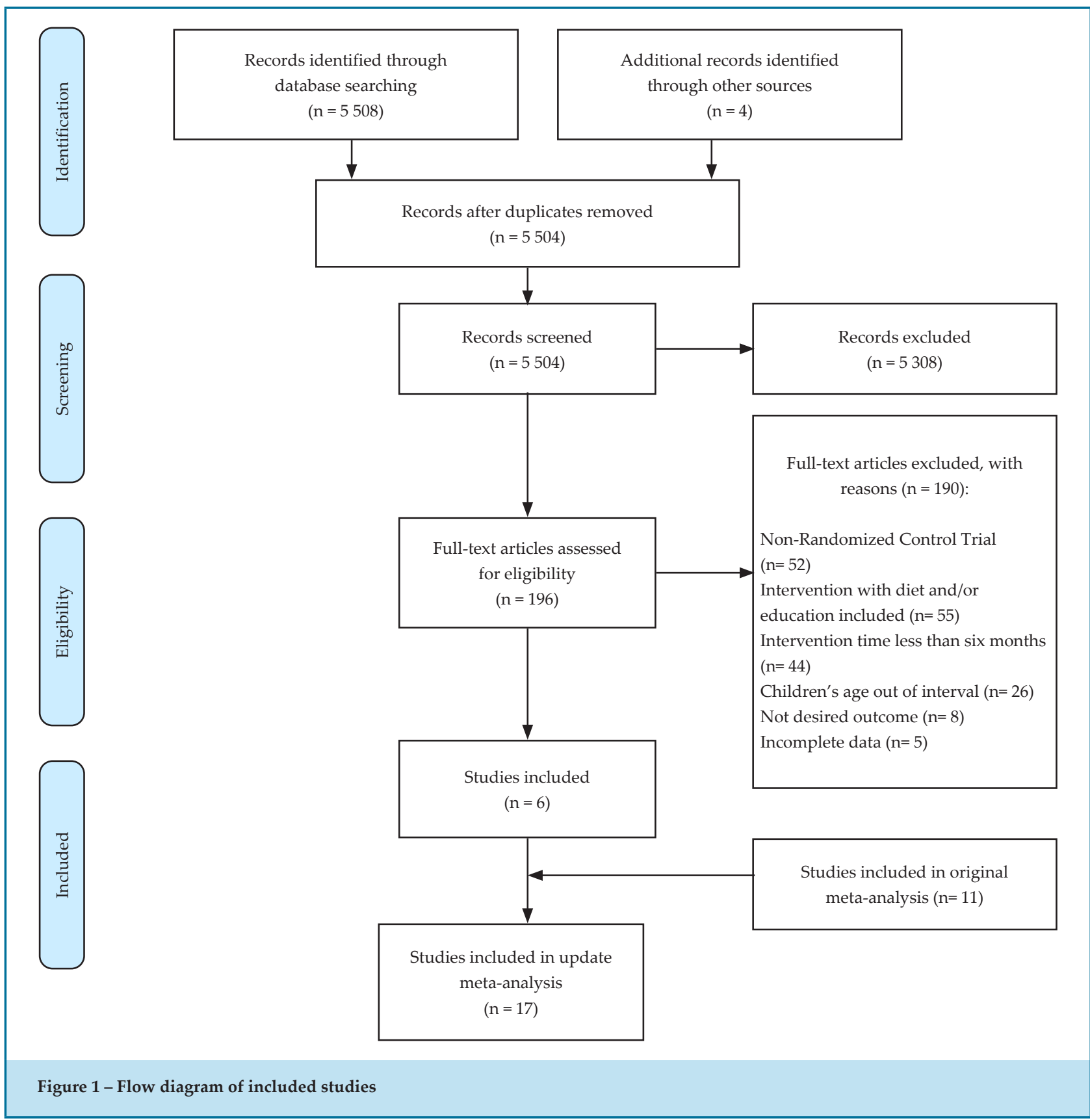

\section{Blood pressure}

\section{Systolic and diastolic blood pressure}

Six studies [Vandongen et al., ${ }^{19}$ Kriemler et al., ${ }^{22}$ Walther et al., ${ }^{34}$ Jones et al., ${ }^{20}$ Muller et al., ${ }^{33}$ AguilarCordero et al., ${ }^{35} \mathrm{n}=1,489$, evaluated SBP and five studies [Vandongen et al., ${ }^{19}$ Kriemler et al. ${ }^{22}$ Walther et al., ${ }^{34}$ Jones et al., ${ }^{30}$ and Aguilar-Cordero et al., ${ }^{36} \mathrm{n}=1,170$, evaluated DBP. Physical activity interventions were associated with reductions in SBP and DBP when compared to less intensive physical activity interventions or no intervention (Figure 4). These results are not different from the previous data. ${ }^{10}$

\section{Discussion}

The present study is the first update, since 2014, of the systematic review with meta-analysis including RCTs in children submitted to interventions involving physical exercises of at least six months' duration, differing from other systematic reviews ${ }^{36,37}$ published 


\section{Appendix III - Table 1 - Characteristics of included studies ${ }^{1}$}

\begin{tabular}{|c|c|c|c|c|c|c|}
\hline Study, year & $\begin{array}{c}\text { Randomized } \\
\text { patients (n) } \\
\text { intervention/ } \\
\text { control }\end{array}$ & Participants & Intervention group & $\begin{array}{l}\text { Control } \\
\text { group }\end{array}$ & $\begin{array}{c}\text { Intervention } \\
\text { duration }\end{array}$ & Outcome measures \\
\hline $\begin{array}{l}\text { Vandongen } \\
\text { et al., } 1995\end{array}$ & $158 / 145$ & $\begin{array}{l}\text { School } \\
\text { children }\end{array}$ & $\begin{array}{l}\text { Six times per week (30 } \\
\text { minutes } \\
\text { each) classroom sessions } \\
\text { replacing the usual } \\
\text { curriculum }\end{array}$ & $\begin{array}{c}\text { Regular } \\
\text { curriculum }\end{array}$ & 9 months & $\begin{array}{l}\text { Body mass index, } \\
\text { systolic blood } \\
\text { pressure, diastolic } \\
\text { blood pressure, total } \\
\text { cholesterol, } \\
\text { triglycerides }\end{array}$ \\
\hline
\end{tabular}

\begin{tabular}{|c|c|c|c|c|c|c|}
\hline $\begin{array}{l}\text { Barbeau et } \\
\text { al., 2007; }\end{array}$ & $118 / 83$ & $\begin{array}{c}\text { School } \\
\text { children } \\
\text { (black girls) }\end{array}$ & $\begin{array}{l}\text { Physical education classes } \\
\text { plus } 110 \text { minutes of out- } \\
\text { of-school physical activity }\end{array}$ & $\begin{array}{c}\text { Regular } \\
\text { physical } \\
\text { education } \\
\text { classes }\end{array}$ & 1 school year & Body mass index \\
\hline $\begin{array}{l}\text { Weintraub et } \\
\text { al., } 2008\end{array}$ & $09 / 12$ & $\begin{array}{l}\text { School } \\
\text { children }\end{array}$ & $\begin{array}{c}\text { Out-of-school sports } \\
\text { program }\end{array}$ & $\begin{array}{l}\text { Traditional } \\
\text { health } \\
\text { education }\end{array}$ & 6 months & Body mass index \\
\hline
\end{tabular}

\begin{tabular}{|c|c|c|c|c|c|c|}
\hline $\begin{array}{l}\text { Donnelly et } \\
\text { al., } 2009\end{array}$ & $814 / 713$ & $\begin{array}{c}\text { School } \\
\text { children } \\
\text { and parents }\end{array}$ & $\begin{array}{l}\text { Physical education classes } \\
\text { ( } 60 \text { minutes per week) } \\
\text { plus } 90 \text { minutes per week } \\
\text { of moderate to vigorous } \\
\text { physically active academic } \\
\text { lessons (3.0-6.0 metabolic } \\
\text { equivalents of task (MET), } \\
10 \text { min each) delivered } \\
\text { intermittently throughout } \\
\text { the school day }\end{array}$ & $\begin{array}{l}\text { Regular } \\
\text { physical } \\
\text { education } \\
\text { classes }\end{array}$ & 3 years & Body mass index \\
\hline $\begin{array}{l}\text { Walther et } \\
\text { al., } 2009\end{array}$ & $105 / 56$ & $\begin{array}{c}\text { School } \\
\text { children } \\
\text { and parents }\end{array}$ & $\begin{array}{c}45 \text { minutes of physical } \\
\text { education classes per } \\
\text { school day }\end{array}$ & $\begin{array}{l}\text { Two physical } \\
\text { education } \\
\text { classes per } \\
\text { week } \\
\text { (45 minutes } \\
\text { each) }\end{array}$ & 1 school year & $\begin{array}{c}\text { Total cholesterol, } \\
\text { triglycerides }\end{array}$ \\
\hline $\begin{array}{l}\text { Faude et al., } \\
2010\end{array}$ & $11 / 11$ & $\begin{array}{c}\text { Overweight } \\
\text { children }\end{array}$ & $\begin{array}{c}\text { One hour football } \\
\text { training } 3 \text { times per week } \\
\text { (1 hour each })\end{array}$ & $\begin{array}{l}\text { Standard } \\
\text { sports } \\
\text { program }\end{array}$ & 6 months & Body mass index \\
\hline $\begin{array}{l}\text { Kriemler et } \\
\text { al., } 2010\end{array}$ & $297 / 205$ & $\begin{array}{l}\text { School } \\
\text { children }\end{array}$ & $\begin{array}{c}\text { Five physical education } \\
\text { lessons per week }\end{array}$ & $\begin{array}{c}\text { Three } \\
\text { physical } \\
\text { education } \\
\text { lessons per } \\
\text { week }\end{array}$ & 12 months & $\begin{array}{l}\text { Body mass index, } \\
\text { systolic blood } \\
\text { pressure, diastolic } \\
\text { blood pressure, } \\
\text { total cholesterol, } \\
\text { triglycerides }\end{array}$ \\
\hline Li et al., 2010 & $2329 / 2371$ & $\begin{array}{l}\text { School } \\
\text { children }\end{array}$ & $\begin{array}{c}\text { Two daily } 10-\mathrm{min} \\
\text { physical activity sessions } \\
\text { conducted in the break } \\
\text { between classes }\end{array}$ & $\begin{array}{c}\text { No } \\
\text { intervention. } \\
\text { (Regular } \\
\text { physical } \\
\text { education } \\
\text { classes } \\
\text { and after- } \\
\text { school } \\
\text { activities) }\end{array}$ & 1 school year & Body mass index \\
\hline
\end{tabular}




\begin{tabular}{|c|c|c|c|c|c|c|}
\hline $\begin{array}{l}\text { Jansen et al., } \\
2011\end{array}$ & $1240 / 1382$ & $\begin{array}{l}\text { School } \\
\text { children }\end{array}$ & $\begin{array}{l}\text { Three physical education } \\
\text { classes per week plus } \\
\text { additional sports and } \\
\text { play activities outside } \\
\text { school hours (voluntary) }\end{array}$ & $\begin{array}{l}\text { Two physical } \\
\text { education } \\
\text { classes }\end{array}$ & 8 months & Body mass index \\
\hline $\begin{array}{l}\text { Thivel et al., } \\
2011\end{array}$ & $229 / 228$ & $\begin{array}{l}\text { Lean and } \\
\text { obese } \\
\text { school } \\
\text { children }\end{array}$ & $\begin{array}{l}\text { Two physical education } \\
\text { classes per week plus } \\
2 \text { times additional } \\
\text { exercise classes ( } 2 \text { times, } \\
60 \text { minutes each = } 120 \\
\text { minutes per week) }\end{array}$ & $\begin{array}{l}\text { Two physical } \\
\text { education } \\
\text { classes per } \\
\text { week }\end{array}$ & 6 months & Body mass index \\
\hline $\begin{array}{l}\text { Walther et } \\
\text { al., } 2011\end{array}$ & $141 / 91$ & $\begin{array}{l}\text { School } \\
\text { children }\end{array}$ & $\begin{array}{l}\text { One hour of regulated } \\
\text { sports activities, } \\
\text { including } 15 \text { minutes of } \\
\text { endurance training five } \\
\text { times a week }\end{array}$ & $\begin{array}{l}\text { One hour of } \\
\text { current sports } \\
\text { activity two } \\
\text { times a week }\end{array}$ & 2 school years & $\begin{array}{l}\text { Systolic blood } \\
\text { pressure, } \\
\text { diastolic blood } \\
\text { pressure }\end{array}$ \\
\hline $\begin{array}{l}\text { Martínez- } \\
\text { Vizcaíno et } \\
\text { al., } 2014\end{array}$ & $420 / 492$ & $\begin{array}{l}\text { School } \\
\text { children }\end{array}$ & $\begin{array}{l}\text { Two 90-minute physical } \\
\text { activity sessions during } \\
\text { the weekdays in the } \\
\text { evening from } 4 \text { to } 5.30 \\
\text { pm and one 150-minute } \\
\text { session on Saturday } \\
\text { morning each week }\end{array}$ & $\begin{array}{l}\text { Conventional } \\
\text { physical } \\
\text { education } \\
\text { curriculum }\end{array}$ & 9 months & Body mass index \\
\hline $\begin{array}{l}\text { Sacchetti et } \\
\text { al., } 2014\end{array}$ & $212 / 216$ & $\begin{array}{l}\text { School } \\
\text { children }\end{array}$ & $\begin{array}{l}\text { 30-minute physical } \\
\text { exercise daily }\end{array}$ & $\begin{array}{l}\text { Regular } \\
\text { physical } \\
\text { education } \\
\text { classes }\end{array}$ & 2 years & Body mass index \\
\hline $\begin{array}{l}\text { Naiman et } \\
\text { al., } 2014\end{array}$ & $110 / 110$ & $\begin{array}{l}\text { School } \\
\text { children }\end{array}$ & $\begin{array}{l}\text { Two hours of out-of- } \\
\text { school physical activities } \\
\text { five times per week }\end{array}$ & $\begin{array}{l}\text { Regular } \\
\text { after school } \\
\text { routine }\end{array}$ & 9 months & Body mass index \\
\hline $\begin{array}{l}\text { Muller et al., } \\
2015\end{array}$ & $202 / 164$ & $\begin{array}{l}\text { School } \\
\text { children }\end{array}$ & $\begin{array}{l}60 \text { minutes of physical } \\
\text { exercise at school daily ( } 5 \\
\text { hours per week) }\end{array}$ & $\begin{array}{l}\text { Two physical } \\
\text { education } \\
\text { classes (45 } \\
\text { minutes } \\
\text { each) weekly }\end{array}$ & 4 years & $\begin{array}{c}\text { Systolic blood } \\
\text { pressure, low- } \\
\text { density lipoprotein } \\
\text { cholesterol, } \\
\text { high-density } \\
\text { lipoprotein cholesterol, } \\
\text { triglycerides }\end{array}$ \\
\hline $\begin{array}{l}\text { Jones et al, } \\
2015\end{array}$ & $19 / 18$ & $\begin{array}{l}\text { School } \\
\text { children }\end{array}$ & $\begin{array}{l}90 \text { minutes of physical } \\
\text { exercise twice a week }\end{array}$ & 45 min week & 7 months & $\begin{array}{c}\text { Body mass index, } \\
\text { total cholesterol, low- } \\
\text { density lipoprotein } \\
\text { cholesterol, } \\
\text { high-density } \\
\text { lipoprotein cholesterol }\end{array}$ \\
\hline
\end{tabular}

\section{.}

\section{.}

Jansen et al.,

Thivel et al.

Walther et

Vizcaíno et

al., 2014

Sacchetti et

Naiman et

Muller et al.

2015

$202 / 16$

physical

ise at school daily

hours per week)
Two physical

ducation

classes

wo physical

education

asses pe

week
One hour of

current sport

activity two

times a week diastolic blood

pressure 


\section{Table 2 - Risk of bias of included studies}

\begin{tabular}{|c|c|c|c|c|c|c|c|c|}
\hline $\begin{array}{l}\text { Study, } \\
\text { year }\end{array}$ & $\begin{array}{l}\text { Adequate } \\
\text { sequence } \\
\text { generation }\end{array}$ & $\begin{array}{c}\text { Allocation } \\
\text { concealment }\end{array}$ & $\begin{array}{l}\text { Blinding of } \\
\text { investigator }\end{array}$ & $\begin{array}{c}\text { Blinding } \\
\text { of } \\
\text { participant }\end{array}$ & $\begin{array}{l}\text { Blinding } \\
\text { of } \\
\text { assessors }\end{array}$ & $\begin{array}{l}\text { Blinding } \\
\text { of } \\
\text { outcome } \\
\text { assessors }\end{array}$ & $\begin{array}{c}\text { Intention- } \\
\text { to-treat } \\
\text { analysis }\end{array}$ & $\begin{array}{c}\text { Description } \\
\text { of losses } \\
\text { and } \\
\text { exclusions }\end{array}$ \\
\hline $\begin{array}{l}\text { Vandongen } \\
\text { et al., } 1995\end{array}$ & $\begin{array}{c}\text { Not } \\
\text { reported }\end{array}$ & Not reported & Not reported & $\begin{array}{c}\text { Not } \\
\text { reported }\end{array}$ & Yes & Unclear & No & Yes \\
\hline $\begin{array}{l}\text { Barbeau et } \\
\text { al., } 2007\end{array}$ & $\begin{array}{c}\text { Not } \\
\text { reported }\end{array}$ & Unclear & Not reported & $\begin{array}{c}\text { Not } \\
\text { reported }\end{array}$ & $\begin{array}{l}\text { Not } \\
\text { reported }\end{array}$ & $\begin{array}{c}\text { Not } \\
\text { reported }\end{array}$ & Yes & Yes \\
\hline $\begin{array}{l}\text { Weintraub } \\
\text { et al., } 2008\end{array}$ & Yes & Unclear & No & No & No & $\begin{array}{c}\text { Not } \\
\text { reported }\end{array}$ & Yes & Yes \\
\hline $\begin{array}{l}\text { Donnelly et } \\
\text { al., } 2009\end{array}$ & $\begin{array}{c}\text { Not } \\
\text { reported }\end{array}$ & Unclear & No & No & Yes & $\begin{array}{l}\text { Not } \\
\text { reported }\end{array}$ & $\begin{array}{l}\text { Not } \\
\text { reported }\end{array}$ & Yes \\
\hline $\begin{array}{l}\text { Walther et } \\
\text { al., } 2009\end{array}$ & $\begin{array}{c}\text { Not } \\
\text { reported }\end{array}$ & Unclear & No & No & Yes & $\begin{array}{c}\text { Not } \\
\text { reported }\end{array}$ & Yes & Yes \\
\hline $\begin{array}{l}\text { Faude et } \\
\text { al., } 2010\end{array}$ & Yes & Not reported & Not reported & $\begin{array}{c}\text { Not } \\
\text { reported }\end{array}$ & $\begin{array}{c}\text { Not } \\
\text { reported }\end{array}$ & $\begin{array}{l}\text { Not } \\
\text { reported }\end{array}$ & No & Yes \\
\hline $\begin{array}{l}\text { Kriemler et } \\
\text { al., } 2010\end{array}$ & Yes & Adequate & No & No & Yes & $\begin{array}{c}\text { Not } \\
\text { reported }\end{array}$ & Yes & Yes \\
\hline $\begin{array}{l}\text { Li et al., } \\
2010\end{array}$ & Yes & Not reported & Not reported & $\begin{array}{c}\text { Not } \\
\text { reported }\end{array}$ & $\begin{array}{c}\text { Not } \\
\text { reported }\end{array}$ & $\begin{array}{c}\text { Not } \\
\text { reported }\end{array}$ & No & Yes \\
\hline $\begin{array}{l}\text { Jansen et } \\
\text { al., } 2011\end{array}$ & Yes & Not reported & No & No & No & No & Yes & No \\
\hline $\begin{array}{l}\text { Thivel et } \\
\text { al., } 2011\end{array}$ & $\begin{array}{c}\text { Not } \\
\text { reported }\end{array}$ & Not reported & Not reported & Yes & $\begin{array}{c}\text { Not } \\
\text { reported }\end{array}$ & $\begin{array}{c}\text { Not } \\
\text { reported }\end{array}$ & Yes & Yes \\
\hline $\begin{array}{l}\text { Walther et } \\
\text { al., } 2011\end{array}$ & $\begin{array}{c}\text { Not } \\
\text { reported }\end{array}$ & Adequate & No & No & No & $\begin{array}{c}\text { Not } \\
\text { reported }\end{array}$ & No & Yes \\
\hline $\begin{array}{l}\text { Martínez- } \\
\text { Vizcaíno et } \\
\text { al., } 2014\end{array}$ & Yes & Adequate & No & $\begin{array}{c}\text { Not } \\
\text { reported }\end{array}$ & No & $\begin{array}{l}\text { Not } \\
\text { reported }\end{array}$ & Yes & Yes \\
\hline $\begin{array}{l}\text { Sacchetti et } \\
\text { al., } 2014\end{array}$ & $\begin{array}{l}\text { Not } \\
\text { reported }\end{array}$ & Not reported & No & $\begin{array}{c}\text { Not } \\
\text { reported }\end{array}$ & $\begin{array}{c}\text { Not } \\
\text { reported }\end{array}$ & $\begin{array}{c}\text { Not } \\
\text { reported }\end{array}$ & Yes & Yes \\
\hline $\begin{array}{l}\text { Naiman et } \\
\text { al., } 2014\end{array}$ & $\begin{array}{c}\text { Not } \\
\text { reported }\end{array}$ & Adequate & No & No & No & No & Yes & Yes \\
\hline $\begin{array}{l}\text { Muller et } \\
\text { al., } 2015\end{array}$ & $\begin{array}{l}\text { Not } \\
\text { reported }\end{array}$ & Not reported & Not reported & $\begin{array}{c}\text { Not } \\
\text { reported }\end{array}$ & $\begin{array}{c}\text { Not } \\
\text { reported }\end{array}$ & $\begin{array}{c}\text { Not } \\
\text { reported }\end{array}$ & Yes & Yes \\
\hline $\begin{array}{l}\text { Jones et al, } \\
2015\end{array}$ & Yes & Adequate & No & $\begin{array}{c}\text { Not } \\
\text { reported }\end{array}$ & Yes & $\begin{array}{c}\text { Not } \\
\text { reported }\end{array}$ & Yes & Yes \\
\hline $\begin{array}{l}\text { Aguilar- } \\
\text { Cordero et } \\
\text { al., } 2019\end{array}$ & Yes & Adequate & No & No & $\begin{array}{c}\text { Not } \\
\text { reported }\end{array}$ & $\begin{array}{c}\text { Not } \\
\text { reported }\end{array}$ & Yes & Yes \\
\hline
\end{tabular}

after the original meta-analysis. ${ }^{10}$ As in the original publication, we found that the interventions proposed in the RCTs included in this update were not associated with any change in the BMI. Data were extracted from four of the five new articles involving BMI measures included in the update. Muller et al., ${ }^{33}$ used BMI in percentile and not in $\mathrm{kg} / \mathrm{m}^{2}$, so their study was not included. 


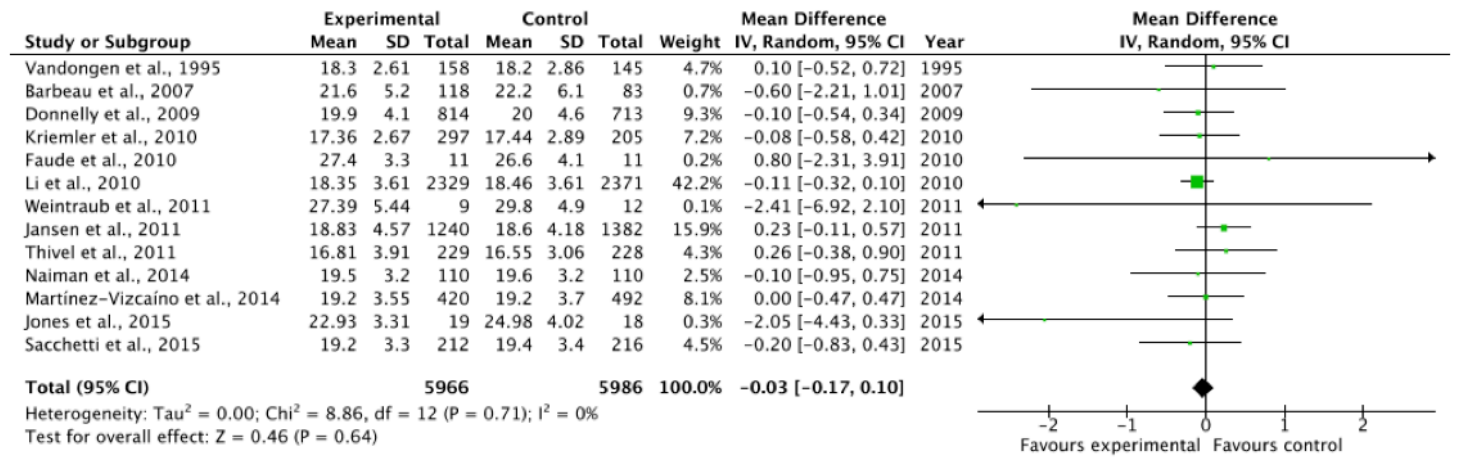

Figure 2 - Absolute changes in body mass index (BMI, $\left.\mathrm{kg} / \mathrm{m}^{2}\right)$ for physical activity intervention vs. controls.

CI: confidence interval; SD: standard deviation. Weights are from random-effects analysis.

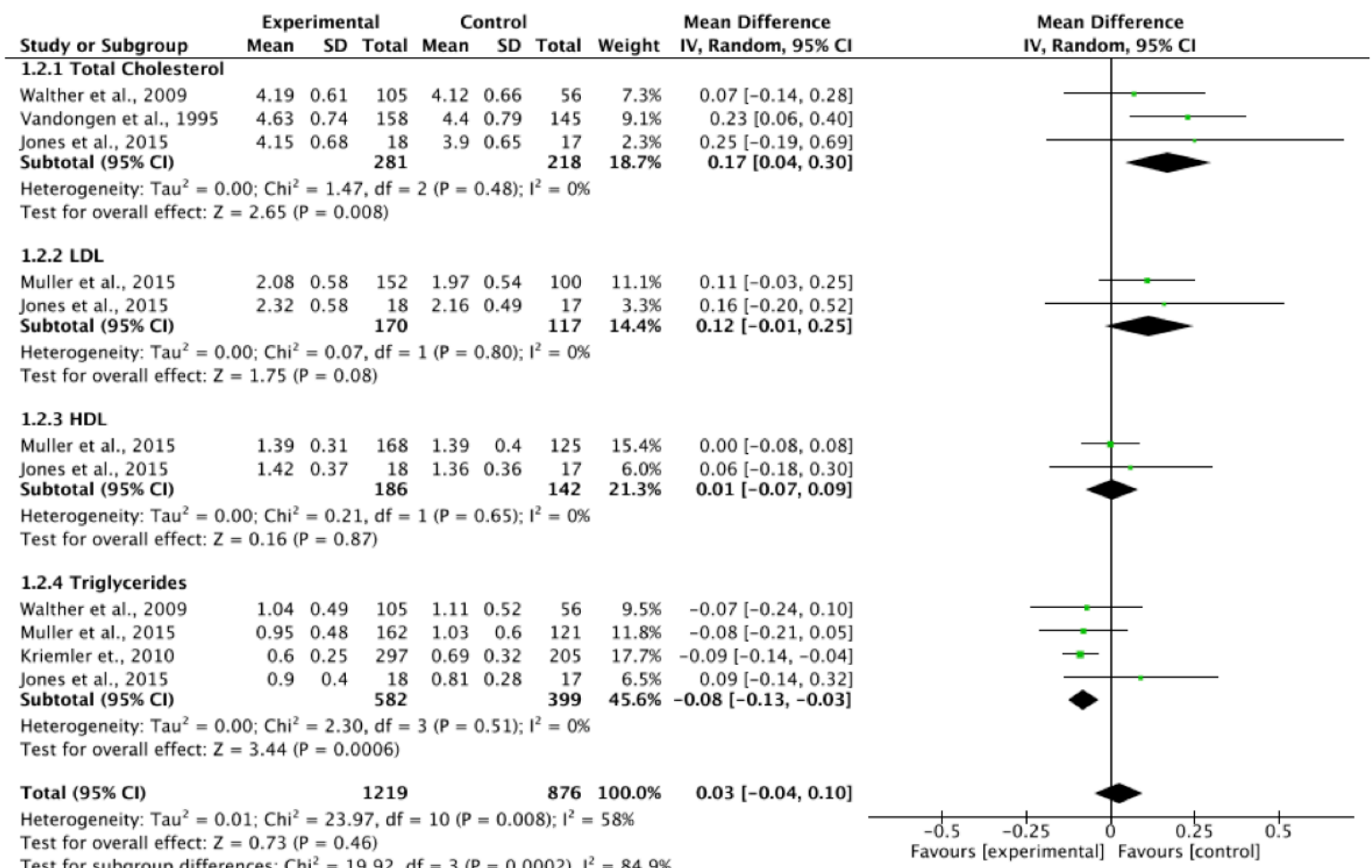

Figure 3 - Absolute changes in total cholesterol, LDL-c, HDL-c and triglycerides (mmol/L) for physical activity intervention vs. controls. CI: confidence interval; SD: standard deviation. Weights are from random-effects analysis.

Brannsether et al., ${ }^{38}$ evaluated differences in BMI in children aged 0-19 years for one year (The Bergen Growth Study). BMI increased slightly and with small variations during childhood, reaching the highest values at 13 years of age and decreasing in older children (the number of children aged 6-14 years was 1,167). It was pointed out that there was a higher prevalence of overweight and obesity in children aged 7-11 years and that these 


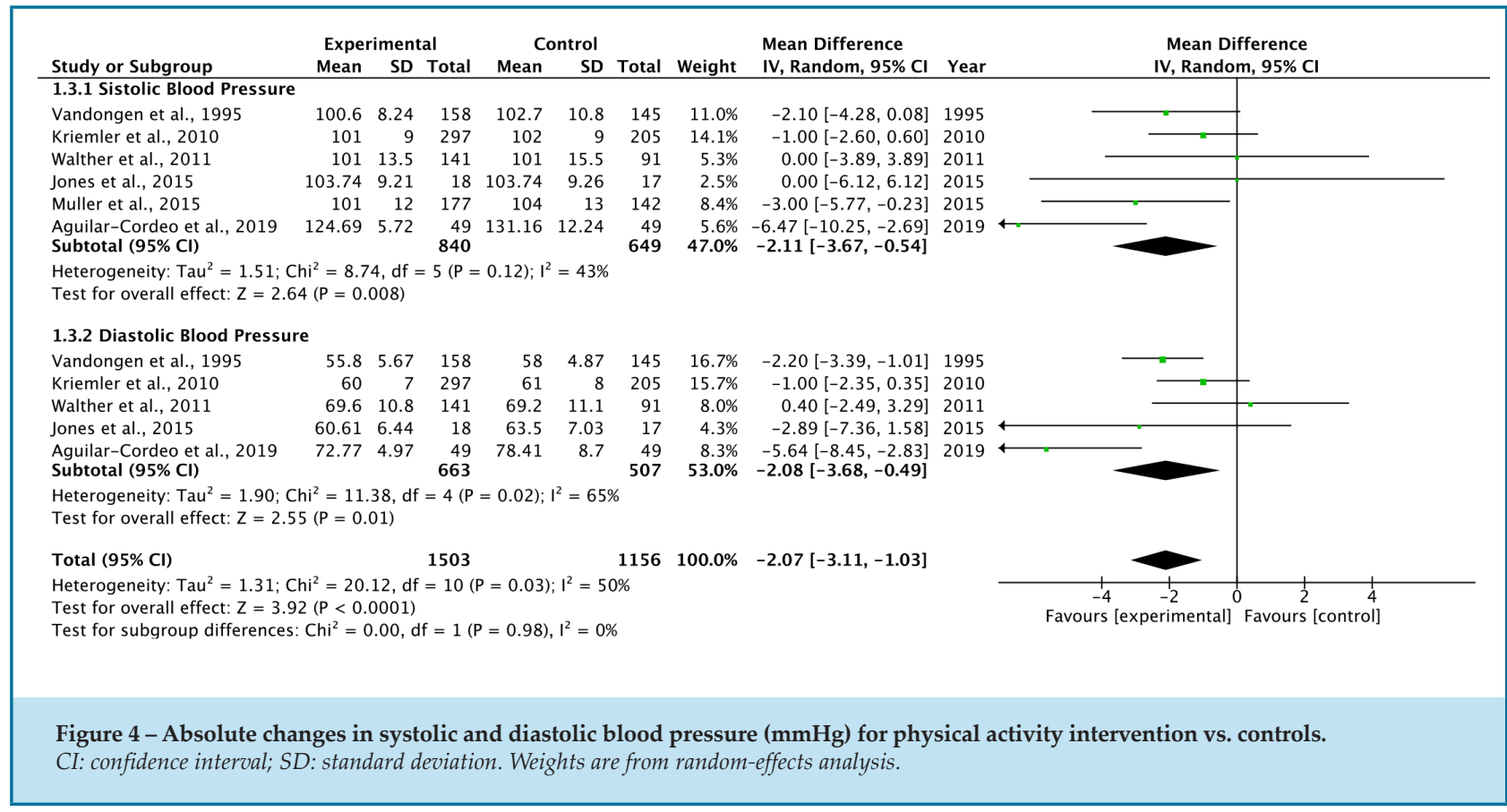

children may, for a period, be taller than those with adequate weight. Therefore, the relationship between weight and height does not change significantly until the child's growth rate decreases. In line with our original publication, it is necessary to reaffirm that $\mathrm{BMI}$ is not the best parameter to measure the effect of interventions performed in the clinical trials of this systematic review, precisely because an increase in the index is expected during the studied age range. In addition, only four new studies were added the original analysis of BMI, adding a total of 1,597 children to that of the original publication ( $n=10,355)$. Thus, the observations made in the original review that there was little difference between the control and intervention groups are still valid, since all control groups received regular physical education classes whereas the intervention groups underwent additional exercise sessions. Moreover, to obtain more powerful results in reducing weight in overweight and obese children it is of utmost importance the combination of nutritional interventions (healthier eating habits and choices, lower caloric intake, etc). In this regard, Verjans-Janssen et al. conducted a systematic review of 18 studies where different approaches (physical activity, nutrition, diet, parental involvement and others) were used; 11 studies showed favorable results for BMI, six reported no change and one showed a negative outcome. ${ }^{39}$ That review brought results of BMI's reduction, but, again, the review includes studies on combinations of multiple interventions, which differs from the current study where only studies on single physical activity interventions were included.

In addition, the present investigation corroborated the inverse relationship between blood pressure and physical activity in children and adolescents, as presented in our first review ${ }^{11}$ and other studies..$^{40,41}$ Normal levels of blood pressure were expected as a result of improvement in cardiorespiratory fitness. ${ }^{40}$ We may hypothesize that this positive effect may be carried into adulthood, and contribute to cardiovascular risk prevention, since we know that these risk factors (physical inactivity, increased blood pressure and excess weight) start in childhood and may persist throughout life. Furthermore, these positive findings may also indicate a protective role of these factors for hypertension in adults. ${ }^{41}$ Liu et al., ${ }^{42}$ in a longitudinal cohort study with adult participants in the Bogalusa Heart Study, who had been followed since childhood, demonstrated that the increase in BMI in childhood and adulthood and its overload throughout life are significantly associated with arterial stiffness in young adults, triggered by an increasing trend of hypertension. ${ }^{42}$

In 2014, we reported that more physical activity in children was associated with a reduction in TG and an increase in TC. ${ }^{11}$ For the new included outcomes LDL-c and HDL-c, the RCTs analyzed in this review did not 
show significant results, which can be justified by the small number of individuals evaluated. The only lipid component that showed positive repercussions after intervention with physical activity was TG. These results are in line with another meta-analysis that evaluated physical activity intervention in overweight children and adolescents. ${ }^{43}$ High levels of TG are an independent risk factor for coronary heart disease, for their potential atherogenic effects. ${ }^{44}$ Thus, the results demonstrated in the present study corroborate the importance of the practice of physical activity as a non-pharmacological measure to control elevations in TG levels. Unlike our findings, another systematic review with meta-analysis ${ }^{45}$ involving children and adolescents between two and 17 years of age, demonstrated that exercise is associated with LDL-c reduction. However, the authors pointed out that greater reductions in LDL-c were associated with an older age of the adolescents, which could justify the difference in relation to our study, where children between 6 and 12 years were included.

The conflicting results between physical exercise and repercussions on HDL-c levels were also highlighted in another systematic review. ${ }^{46}$ Stoner et al., ${ }^{47}$ carried out a meta-analysis to assess the effects of physical exercise on cardiometabolic risk factors in adolescents with overweight or obesity and demonstrated no changes in HDL-c levels, in accordance with the present study. Longer periods of intervention with physical activity may be necessary, and aerobic exercise is more beneficial in comparison to regular strength training to improve blood lipids. ${ }^{47}$ In disagreement with the present study, Costa et al., ${ }^{45}$ demonstrated in a meta-analysis that supervised physical training was associated with a reduction in TC levels compared to no intervention. However, the authors showed that the longer the duration of the follow-up, the smaller the reductions, due to the adaptation of the TC levels. This inverse association could justify the absence of changes in TC levels in the present study, considering that only interventions of at least six months' duration were included. In addition, it is well-known that the peak of TC curves in healthy children aged between 9 and 10 years is approximately $15 \mathrm{mg} / \mathrm{dL}$ higher than in younger children and adolescents. ${ }^{48}$ Considering that in the study by Costa et al. ${ }^{45}$ the sample had a wider age range (between 2 and 17 years), it may not have been sensitive to possible associations between age groups and borderline cholesterol values .
This systematic review has limitations due to the poor methodological quality of the included studies. The absence of blinding can be considered one of the greatest limitations among these studies, although it is usual in non-pharmacological studies. Studies that involve physical activities and no pharmacological intervention have limitations regarding learning curves, introduction and stabilization of the intervention, and combination of other interventions that do not assess physical activity. However, the interventions used in the selected papers were similar, providing strength for the analysis of several components even in a small sample. In our first ${ }^{10}$ and in the current systematic review, several studies have been excluded due to the lack of description of the interventions, resulting in a reduced number of RCTs included and of outcomes analyzed. However, considering that these physical activity interventions are of low cost and risk, the positive findings, in favor of the interventions, should be worthy of consideration even in a small sample.

Finally, this systematic review with meta-analysis has strengths that should be mentioned. The process of article selection was conducted with strict criteria, namely RCTs, studies with an intervention period longer than six months (27 weeks), and with outcomes already mentioned in our previous systematic review additionally to HDL-c and LDL-c. In addition, children and adolescents were included regardless of baseline BMI, and the original language of the studies was not considered as an exclusion criterion.

\section{Conclusions}

This update confirms the previous findings of the beneficial effects of physical activity interventions of at least six months' duration in reducing SBP, DBP, as well as TG levels. Although no changes in other cardiovascular risk factors (BMI, TC, HDL-c and LDL-c) were detected, we believe that regular physical activity classes in schools must still be encouraged as a way of preventing diseases and promoting health for children and adolescents, primarily during pubertal development and, later, for consolidation of healthy habits throughout life.

\section{Acknowledgements}

We were thankful for general support of National Council for Scientific and Technological Development - 
CNPq, Rio Grande do Sul Research Foundation (Fundação de Apoio à Pesquisa do Estado do Rio Grande do Sul - FAPERGS) and Institute of Cardiology Research Foundation (Fundo de Apoio do Instituto de Cardiologia/FUC à Ciência e Cultura - FAPICC).

\section{Author contributions}

Conception and design of the research: Cesa CC, Barbiero S, Schaan BD, Pellanda LC. Acquisition of data: Cesa CC, Molino G, Lima J, Pereira R, Eibel B, Barbiero S, Pellanda LC. Analysis and interpretation of the data: Cesa CC, Molino G, Lima J, Pereira R, Eibel B, Barbiero S, Schaan BD, Pellanda LC. Statistical analysis: Cesa CC. Writing of the manuscript: Cesa CC, Molino G, Lima J, Pereira R, Eibel B, Barbiero S, Pellanda LC. Critical revision of the manuscript for intellectual content: Cesa CC, Molino G, Lima J, Pereira R, Eibel B, Barbiero S, Schaan BD, Pellanda LC.

\section{References}

1. Kumar S, Kelly AS. Review of Childhood Obesity: From Epidemiology, Etiology, and Comorbidities to Clinical Assessment and Treatment. Mayo Clin Proc. 2017;92(2):251-65.

2. Di Cesare M, Soric M, Bovet P, Miranda J, Bhutta Z, Stevens GA, et al. The Epidemiological Burden of Obesity in Childhood: A Worldwide Epidemic Requiring Urgent Action. BMC Med. 2019;17(1):212

3. Lee EY, Yoon KH. Epidemic Obesity in Children and Adolescents: Risk Factors and Prevention. Front Med. 2018;12(6):658-66.

4. Bjerregaard LG, Jensen BW, Angquist L, Osler M, Sorensen T, Baker JL, et al. Change in Overweight From Childhood to Early Adulthood and Risk of Type 2 Diabetes. N Engl J Med. 2018;378(14):1302-12.

5. Weihrauch-Blüher S,Schwarz $\mathrm{P}<$ Klussman JH. Childhood obesity: increased risk for cardiometabolic disease and cancer in adulthood. Metabolism. 2019;92:147-52.

6. Seabra A, Katzmarzyk P,Carvalho MJ, Coelho-E-Silva M, Abreu S, et al. Effects of 6-month Soccer and Traditional Physical Activity Programmes on Body Composition, Cardiometabolic Risk Factors, Inflammatory, Oxidative Stress Markers and Cardiorespiratory Fitness in Obese Boys. J Sports Sci. 2016;34(19):1822-9.

7. Ozemek C,Ladelu DR, Lavie CJ, Clayeis H, Kaminsky LA, Rodd R, et al. An Update on the Role of Cardiorespiratory Fitness, Structured Exercise and Lifestyle Physical Activity in Preventing Cardiovascular Disease and Health Risk Prog Cardiovasc Dis. 2018;61(5-6):484-90 .

8. Lear SA, Rangarajan S, Gavevic D, Leong D, Iqbal R, Casanova A, et al. The Effect of Physical Activity on Mortality and Cardiovascular Disease in 130000 People From 17 High-Income, Middle-Income, and LowIncome Countries: The PURE Study. Lancet. 2017;390(10113):2643-54.

9. Davis CL, Litwin SE, Pollock NK, Waller JL, Zhu H, Dong Y, et al. Exercise effects on arterial stiffness and heart health in children with excess weight: The SMART RCT. Int J Obes (Lond). 2020; 44(5):1152-63.

10. Cesa CC, Sbruzzi G, Ribeiro RA, Barbiero SM, Petrowicx RO, Machado N, et al. Physical Activity and Cardiovascular Risk Factors in Children: Meta-Analysis of Randomized Clinical Trials. Prev Med. 2014;69:54-62.

11. Clarke M, Horton R. Bringing it all together: Lancet-Cochrane collaborate on systematic reviews. Lancet. 2001 Jun 2;357(9270):1728.

\section{Potential Conflict of Interest}

No potential conflict of interest relevant to this article was reported.

\section{Sources of Funding}

There were no external funding sources for this study.

\section{Study Association}

This study is not associated with any thesis or dissertation work.

\section{Ethics approval and consent to participate}

This article does not contain any studies with human participants or animals performed by any of the authors.

12. MoherD, LiberatiA, TetzlaffJ,AltmanDG. PRISMA Group. Preferred reporting items for systematic reviews and meta-analyses: the PRISMA statement . Int J Surg. 2010;8(5):336-41.(correction appears Int J Surg.2010;8(8):658)

13. U.S. Department of Health and Human Services. Physical activity guidelines for Americans. Washington, D.C; 2008.

14. Haskell WL, Lee I.M, Pate RR, Powell KE, Blair SN, Franklin BA, et al. Physical activity and public health: updated recommendation for adults from the American College of Sports Medicine and the American Heart Association. Circulation. 2008; 116(9):1081-93.

15. Robinson KA, Dickersin K. Development of a highly sensitive search strategy for the retrieval of reports of controlled trials using PubMed. Int. J. Epidemiol. 2002;31(1):150-3.

16. Higgins JPT,Savovic J, Page M, Elbers RG, Sterne AC. Assessing risk of bias in non- randomized trial. In: Higgins JPT, Thomas J, Chandler J, Cumpston M, Li T, Page MJ, Welch VA (editors). Cochrane Handbook for Systematic Reviews of Interventions version 6.0 [Cited in July 2019]. Cochrane, 2019. Available from www.training.cochrane.org/handbook.

17. Higgins, J., Green, S. Cochrane handbook for systematic reviews of interventions, 5th ed. Washington, DC; 2008.

18. Higgins JP, Thompson SG, Deeks JJ, Altman DG. Measuring inconsistency in metaanalyses. BMJ. 2003;327(7414):557-60.

19. Vandongen R, Jenner DA, Thompson C, Taggart EE, Spickett EE, Burke V, et al. A controlled evaluation of a fitness and nutrition intervention program on cardiovascular health in 10- to 12-year-old children. Prev Med. 1995;24(1):9-22.

20. Barbeau P, Johnson MH, Howe CA. Allison J, Davis CL. Gutin B, et al. Ten months of exercise improves general and visceral adiposity, bone, and fitness in black girls. Obesity (Silver Spring). 2007;15(8):2077-85.

21. Donnelly JE, Greene JL, Gibson CA, Smith BK, Wasburn RA, Sullivan DK, et al. Physical Activity Across the Curriculum (PAAC): a randomized controlled trial to promote physical activity and diminish overweight and obesity in elementary school children. Prev Med. 2009;49(4):336-41.

22. Kriemler S, Zahner L, Schindler C,Meyer U, Hartman T, Hebestreit H, et al Effect of school based physical activity programme (KISS) on fitness and adiposity in primary schoolchildren: cluster randomised controlled trial. BMJ. 2010; 340: c785. 
23. Li YP, Hu XQ, Schouten EG, Liu AL, Dei SM, Li L-Z. Report on childhood obesity in China (8): effects and sustainability of physical activity intervention on body composition of Chinese youth. Biomed Environ Sci. 2010;23(3):180-7.

24. Faude O, Kerper O, Multhaupt M, Winter C, Beziel K, Meyer T. Football to tackle overweight in children. Scand J Med Sci Sports. 2010;20(Suppl 1):103-10.

25. Jansen W, Borsboom G, Meima A, Jooten - Van Zwanenburg E, Mackenbach JP, Brug J. Effectiveness of a primary school-based intervention to reduce overweight. Int J Pediatr Obes. 2011;6(2-2):e70-e77.

26. Thivel D, Isacco L, Lazaar N, Aucouturier J, Ratel S, Doré E, et al. Effect of a 6-month school-based physical activity program on body composition and physical fitness in lean and obese schoolchildren. Eur J Pediatr.2011;170(11):1435-43.

27. Weintraub DL, Tirumalai EC, Haydel KF, Fujimoto M, Fulton JE, Robinson TN. Team sports for overweight children:the Stanford Sports to Prevent Obesity Randomized Trial (SPORT). Arch Pediatr Adolesc Med. 2008;162(3):232-7.

28. Martínez-Vizcaíno Sanchez-Lopes M, Notario-Pacheco B, SalcedoAguillar F. Gender differences on effectiveness of a school-based physical activity intervention for reducing cardiometabolic risk: a cluster randomized trial. Int J Behav Nutr Phys Activ. 2014;11:154

29. Khan NA, Raine LB, Drollette ES, Scudder MR, Pontifex MB, Castelli DM, et al. Impact of the FITKids physical activity intervention on adiposity in prepubertal children. Pediatrics. 2014;133(4):e875-e883.

30. Jones RA, Kelly J, Cliff DP, Batterham M, Okely AD. Acceptability and Potential Efficacy of Single-Sex After-School Activity Programs for Overweight and At-Risk Children: The Wollongong SPORT RCT. Pediatr Exerc Sci. 2015;27(4):535-45.

31. Sacchetti R, Ceciliani A, Garulli A, Dallolio L, Beltrami P, Leoni E. Effects of a 2-year school-based intervention of enhanced physical education in the primary school. J Sch Health. 2013;83(9):639-46.

32. Walther C, Gaede L, Adams V,Gelbrich G, Leichtle GG, Sonnabend M, et al. Effect of increased exercise in school children on physical fitness and endothelial progenitor cells: a prospective randomized trial. Circulation. 2009;120(22):2251-9.

33. Müller UM, Walther C, Adams V, Mende M, Adam J, Fikenzen K, et al. Long term impact of one daily unit of physical exercise at school on cardiovascular risk factors in school children. Eur J Prev Cardiol. 2015;23(13):1444-52.

34. Walther C, Mende M, Gaede L, Muller U, Machalica K, Schuler G. Effects of daily physical exercise at school on cardiovascular risk-Results of a 2-year cluster-randomized study. Dtsch Med Wochenschr. 2011;136(46):2348-54

35. Aguilar-Cordero MJ, Rodríguez-Blanque R, Leon-Ríos X, Expósito Ruiz M, García García I, Sánchez-López AM. Influence of Physical Activity on Blood Pressure in Children With Overweight/Obesity: A Randomized Clinical Trial. Am J Hypertens. 2019;33(2):131-6.
36. Mead E, Brown T, Rees K, Azevedo L, Whittalter V, Jones D, et al. Diet, physical activity and behavioural interventions for the treatment of overweight or obese children from the age of 6 to 11 years. Cochrane Database Syst Rev. 2017;6(6):CD012651.

37. Bahia L, Schaan CW, Sparrenberger K, Abreu GA, Barufaldi LA, Coutinho $\mathrm{W}$, et al. Overview of meta-analysis on prevention and treatment of childhood obesity. J Pediatr (Rio J). 2019;95(4):385-400.

38. Brannsether B, Eide GE, Roelants M, Bjerknes R, Júlíusson PB. BMI and BMI SDS in childhood: annual increments and conditional change. Ann Hum Biol. 2017;44(1):28-33.

39. Verjans-Janssen SR B, van de Kolk I, Van Kann DHH, Kremers SPJ,Gerards SMP. Effectiveness of school-based physical activity and nutrition interventions with direct parental involvement on children's BMI and energy balance-related behaviors - A systematic review. PLOS ONE. 2018;13(9):e0204560.

40. Arija V, Villalobos F, Pedret R, Viniesa A, Jovani D, et al. Physical activity, cardiovascular health, quality of life and blood pressure control in hypertensive subjects: randomized clinical trial. Health Qual Life Outcomes. 2018;16(1):184.

41. Chen X, Wang Y. Tracking of blood pressure from childhood to adulthood: a systematic review and meta-regression analysis. Circulation. 2008;117(25):3171-80.

42. Liu Y, Yan Y, Yang X, Li S, Bazzano L, He J. et al. Long-Term Burden of Higher Body Mass Index and Adult Arterial Stiffness Are Linked Predominantly Through Elevated Blood Pressure. Hypertension. 2019;73(1):229-34.

43. Kelley GA, Kelley KS. Aerobic exercise and lipids and lipoproteins in children and adolescents: a meta-analysis of randomized controlled trials. Atherosclerosis. 2007;191(2):447-53.

44. Peng J, Luo F, Ruan G, Peng R, Li X. Hypertriglyceridemia and atherosclerosis. Lipids Health Dis. 2017;16(1):233.

45. Costa RR, Barroso BM, Reichert T, Vieira LFM, Kruel LFM Effects of supervised exercise training on lipid profile of children and adolescents: Systematic review, meta-analysis and meta-regression. Science Sports. 2020;35(6):321-9.

46. Fraga A S, Ladeia AMT,Sá CKC, Tenório MCC. Efeito do exercício sobre os níveis de HDL-c: uma revisão sistemática de metanálises. Rev Bras Medi Esporte. 2017; 23(6):488-94.

47. Stoner L, Rowlands D, Morrison A, Credeur D, Hamlin M, Gaffney K, et al. Efficacy of Exercise Intervention for Weight Loss in Overweight and Obese Adolescents: Meta-Analysis and Implications. Sports Med. 2016;46(11):1737-1751.

48. Skinner, AC, Steiner MJ, Chung AE,Perrin EM. Cholesterol curves to identify population norms by Age and Sex in healthy weight children. Clin Pediatr. 2011;51(3):233-7.

\section{*Supplemental Materials}

For additional information, please click here.

For additional information, please click here. 